Annuaire suisse de politique de développement

16 | 1997

Environnement et développement, 5 ans après Rio

\title{
Présentation du dossier
}

\section{(2) OpenEdition}

1 Journals

Édition électronique

URL : http://journals.openedition.org/aspd/803

DOI : 10.4000/aspd.803

ISSN : 1663-9669

Éditeur

Institut de hautes études internationales et du développement

\section{Édition imprimée}

Date de publication : 1 mars 1997

Pagination : 179-180

ISSN : 1660-5934

\section{Référence électronique}

"Présentation du dossier », Annuaire suisse de politique de développement [En ligne], 16 | 1997, mis en ligne le 08 août 2012, consulté le 08 septembre 2020. URL : http://journals.openedition.org/aspd/803 ; DOI : https://doi.org/10.4000/aspd.803

(c) The Graduate Institute I Geneva 


\section{ENVIRONNEMENT ET DÉVELOPPEMENT}

5 ANS APRÈS RIO

P

armi les dossiers qui n'ont pas abouti lors des conférences de l'Uruguay Round, deux revêtent une grande importance pour les pays en développe ment tout en suscitant leur appréhension :

$\checkmark$ celui des relations entre le commerce international et les conditions de travail dans les pays exportateurs, la question dite des « clauses sociales »; $\checkmark$ celui de la place des relations environnementales dans les relations économiques internationales, soit la question de « l'après Rio ».

Nous avons abordé le premier thème dans l'Annuaire Suisse-Tiers Monde 1996 sous le titre «Clauses sociales et commerce Nord-Sud». Nous abordons cette année - en mettant un accent particulier sur la politique de la Suisse dans ce domaine - le rôle que joue la problématique de l'environnement dans les diverses dimensions des relations entre pays industriels et pays en développement. On admet aujourd'hui qu'il est de l'intérêt commun de toutes les nations d'imaginer et de mettre en œuvre un modèle de développement dit « durable » qui assure, à l'échelle mondiale, à la fois une répartition plus équitable du bien-être et la reproduction à long terme de la société humaine.

La transition vers ce modèle se pose en des termes très différents pour les diverses catégories de pays ; il est évident que la Suisse et le Burkina Faso ne saurait avoir à cet égard les mêmes priorités. Pour les pays en développement, il s'agit de veiller à ce que la croissance économique nécessaire pour assurer des niveaux de vie décents à l'ensemble de la population ne contribue pas à aggraver la pression des activités humaines sur les ressources naturelles. Pour les pays industrialisés, il s'agit de trouver un modèle de développement qui permette de réduire la consommation par tête de certaines ressources - les énergies fossiles par exemple - tout en préservant, voire améliorant la qualité de la vie pour l'ensemble de la population. La mise en harmonie de cette double démarche vers le développement « durable » ne va pas sans engendrer des conflits d'intérêts, des malentendus, des suspicions dans les relations Nord-Sud.

Le dossier que nous présentons débute par une contribution de Franco Romerio et Milad Zarin-Nejadan qui fait le point sur les interactions entre environnement et développement et passe en revue les divers instruments financiers, commerciaux et de coopération au développement permettant d'intégrer la dimension environnementale dans les relations Nord-Sud. 
Trois thèmes plus spécifiques sont ensuite abordés :

a) La relation entre commerce international et environnement avec

- un article de Sophie Forster présentant l'état des négociations internationales (au sein de l'OMC, de la CNUCED et de l'OCDE) dans ce domaine ;

- une contribution de René Vossenar sur les effets de l'éco-étiquetage sur l'environnement et le commerce des pays en développement ;

- la présentation par Dorothea Rüesch de la problématique des labels liés aux conditions sociales et écologiques de production des textiles et de l'habillement ; les exemples sont tirés de la situation sur le marché suisse.

b) Le transfert de technologie dans le domaine de l'environnement. Après avoir présenté le concept d' " éco-technologie », Andràs November examine le rôle et les instruments de la coopération avec les pays en développement pour favoriser ce transfert.

c) Enfin, l'article de Michèle Rajaonarivony analyse dans quelle mesure et avec quels résultats, la dimension environnementale a été intégrée à l'important programme de coopération bilatérale entre la Suisse et Madagascar.

La partie « Analyses et positions » comprend en outre un article de Catherine Schümperli Younossian sur le commerce illicite de biens culturels, une dimension aussi ancienne que méconnue des relations Nord-Sud. Cet article fait le point de la situation juridique en Suisse au moment où le Conseil fédéral s'apprête à proposer au Chambres fédérales la ratification d'importantes conventions internationales destinées à lutter contre le trafic illicite d'œuvres d'art. 